\title{
Holographic Duals of Inhomogeneous Systems: The Rainbow Chain and the Sine-Square Deformation Model
}

\author{
Ian MacCormack, ${ }^{1}$ Aike Liu, ${ }^{2}$ Masahiro Nozaki, ${ }^{1}$ and Shinsei Ryu ${ }^{1,3}$ \\ ${ }^{1}$ Kadanoff Center for Theoretical Physics, University of Chicago, Chicago, Illinois 60637, USA \\ ${ }^{2}$ Department of Physics, University of Illinois at Urbana-Champaign, Urbana, Illinois 61801, USA \\ ${ }^{3}$ James Franck Institute, University of Chicago, Chicago, Illinois 6063\%, USA
}

(Dated: December 27, 2018)

\begin{abstract}
A holographic dual description of inhomogeneous systems is discussed. Notably, finite temperature results for the entanglement entropy in both the rainbow chain and the SSD model are obtained holographically by choosing appropriate foliations of the BTZ spacetime. Other inhomogeneous theories are also discussed. The entanglement entropy results are verified numerically, indicating that a wide variety of inhomogeneous field theory phenomenology can be seen in different slicings of asymptotically $A d S_{3}$ spacetimes.
\end{abstract}

\section{CONTENTS}

\section{Introduction}
A. Rainbow chains
B. Möbius and SSD deformations
C. Overview of the paper

II. Different foliations in $\mathrm{AdS} / \mathrm{CFT}$ and entanglement entropy

A. Different foliations in AdS/CFT

1. Rindler evolution

2. Rainbow chain

3. Möbius and SSD evolution

4. Foliations and UV cutoff

5. Solution generating diffeomorphisms (SGDs)

\section{B. Entanglement entropy \\ 1. Zero temperature \\ 2. Finite temperature}

C. CFT on curved spacetime

III. Holographic dual of rainbow chain
A. Defect entanglement at zero temperature
B. Defect entanglement at finite temperature
C. Half-chain entanglement at zero temperature
D. Half-chain entanglement at finite temperature

IV. Holographic dual of SSD
A. Zero temperature
B. Finite temperature
C. Dipolar limit

V. Conclusion

Acknowledgments

A. Application: Particles in a Potential Well

References

\section{INTRODUCTION}

1

2

2

2

3

3

3

3

4

4

5

6

6

6

7

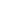

Spatial inhomogeneity is ubiquitous in extended quantum systems (quantum many-body systems) realized, e.g., in solid states and cold atomic gases. One source of inhomogeneity is impurities or randomness, but it can also be introduced intentionally, for example via a harmonic trap confining cold atomic gas. Inhomogeneity can have a dramatic effect in quantum many-body systems, e.g., it can alter the ground states completely - we will discuss some examples momentarily. On the theoretical side, the vast majority of past work has focused on homogeneous systems, and we need to develop new tools to deal with such systems.

In this paper, we study a series of inhomogeneous quantum many-body systems in $(1+1)$ dimensions constructed in the following way: Let us start from a homogeneous quantum many-body Hamiltonian

$$
H=\int d x \mathcal{H}(x)
$$

where $\mathcal{H}$ is the Hamiltonian density, given by the 00 component of the energy-momentum tensor, $\mathcal{H} \sim T_{00}$. The spatial manifold here can be non-compact (i.e., infinite line $x \in[-\infty,+\infty]$ ) or compact (i.e., circle of a finite circumference $L, x \in[0, L])$. Similarly, for a homogeneous system defined on a one-dimensional lattice, one can consider the Hamiltonian $H=\sum_{i} \mathcal{H}_{i}$, where $\mathcal{H}_{i}$ is the Hamiltonian density at a given lattice site $i$. By "deforming" $H$ by an envelope function $f(x)$, we then consider an inhomogeneous system

$$
H[f]=\int d x f(x) \mathcal{H}(x),
$$

or $H[f]=\sum_{i} f\left(x_{i}\right) \mathcal{H}_{i}$ for lattice systems.

Of central focus in this paper are the fundamental properties of the deformed Hamiltonians, mainly the scaling of the entanglement entropy both at zero and finite temperatures. In this work, we will focus on the cases where the original, homogeneous, Hamiltonians are those of $(1+1) \mathrm{d}$ conformal field theories (CFTs). 


\section{A. Rainbow chains}

One deformation of central interest is the so-called rainbow chain. ${ }^{1-6}$ The rainbow chain is an inhomogeneous $(1+1) d$ quantum lattice model, in which the envelope function $f(x)$ decays exponentially,

$$
f(x)=e^{-h|x|},
$$

where $h$ is a parameter. For example, for the free fermion hopping model, we consider

$$
\begin{gathered}
H=\sum_{i, j=1}^{N} t_{i j} c_{i}^{\dagger} c_{j}, \\
t_{i j}=-f_{i} \delta_{i-j, 1}-f_{i} \delta_{i-j,-1},
\end{gathered}
$$

where $c_{i}^{\dagger} / c_{i}$ are the fermion creation/annihilation operators at site $i, N$ is the total number of lattice sites, and

$$
f_{j}=e^{-h|j-N / 2|}
$$

(where the center has been shifted to $j=N / 2$ ). The homogeneous counterpart (where $f_{j}=$ const.) realizes, at half-filling (partial filling), the $c=1$ free fermion CFT in the continuum limit.

An interesting feature of this model is that the entanglement entropy shows volume law scaling; It was found that the entanglement entropy of the reduced density matrix, when the chain is bipartitioned at the center and the half of the chain is traced out, grows linearly with respect to the subsystem size.

It has been also understood that, in the continuum, introducing the rainbow chain deformation is equivalent to putting the CFT on a curved spacetime with the metric: ${ }^{4}$

$$
d s_{A d S_{2}}^{2}=-e^{-2 h|x|} d t^{2}+d x^{2} .
$$

This is the metric of $A d S_{2}$. Here, $h$ is the curvature scale (the inverse radius) of $A d S_{2}$. By the change of the coordinates

$$
\eta=\operatorname{sgn}(x) \frac{e^{h|x|}}{h},
$$

the metric can also be written as

$$
d s_{A d S_{2}}^{2}=\frac{1}{h^{2} \eta^{2}}\left(d \eta^{2}-d t^{2}\right),
$$

which is the Poincaré patch of $A d S_{2}$.

\section{B. Möbius and SSD deformations}

Another interesting class of deformations are Möbius deformations and the sine-square deformation (SSD). ${ }^{7-24}$ Starting from the uniform system defined on a spatial circle of circumference $L$, the Möbius evolution is given by

$$
f(x)=1-\tanh (2 \gamma) \cos \frac{2 \pi x}{L}
$$

Here, $\gamma$ is a parameter; $\gamma=0$ corresponds to the uniform Hamiltonian, whereas when $\gamma \rightarrow \infty$,

$$
f(x)=1-\cos \frac{2 \pi x}{L}=2 \sin ^{2} \frac{\pi x}{L} .
$$

The resulting evolution operator is called the sine-square deformation (SSD) of the original Hamiltonian. Correspondingly, one can consider the lattice Hamiltonian (4), now with the hopping amplitude $f_{j}=2 \sin ^{2}(j \pi / N)$.

The initial interest in the SSD comes from the observation that the ground state of the SSD Hamiltonian on an open chain (when the system is described by CFT), is equal to the ground state of the uniform Hamiltonian on a finite circle with periodic boundary conditions. This feature makes the SSD useful for more efficiently numerically finding the ground state in DMRG.

Similar to the rainbow deformation, the Möbius/SSD deformations can also be understood by putting CFTs on a curved background with the metric ${ }^{22}$

$$
d s_{\text {Mobius }}^{2}=-\left(1-\tanh 2 \gamma \cos \frac{2 \pi x}{L}\right)^{2} d t^{2}+d x^{2} .
$$

\section{Overview of the paper}

In addition to the rainbow and Möbius/SSD deformations, various other examples of inhomogeneous systems include entanglement Hamiltonians, ${ }^{25-27}$ the square root deformation (known in the context of perfect state transfer $),{ }^{20}$ free fermions in harmonic traps and other potentials, ${ }^{28}$ hyperbolic deformations, and others. ${ }^{29-34}$

In this work, we will discuss a series of inhomogeneous $(1+1)$ d systems, which are given as deformations of uniform CFTs. Of particular interest is the scaling of entanglement entropy at zero and finite temperatures, which we obtain by by combining field theory, holographic, and numerical approaches.

As for the holographic approach, we develop holographic duals of inhomogeneous $(1+1) \mathrm{d}$ CFTs by finding appropriate foliations (slicings) of the bulk $A d S_{3}$. The simplest example would be to foliate $A d S_{3}$ by $(1+1) \mathrm{d}$ flat Minkowski spaces, which, at the asymptotic boundary, gives rise to CFT on a flat space. Other foliations are also possible, realizing CFT put on different metrics. For example, $A d S_{3}$ can be foliated by $A d S_{2},{ }^{35-37}$ which, as we will discuss, realizes the rainbow chain at the boundary.

We will also discuss holographic duals of inhomogeneous CFTs at finite temperatures, by starting from the bulk spacetime with the BTZ black hole, and following the same strategy as the zero temperature case mentioned above. We will show that sensible foliations, valid for low temperatures, can easily be constructed by a brute force application of the coordinate transformations that we use to construct the foliations for the corresponding zero-temperature geometry. This naive approach, however, breaks down at higher temperatures, 
and better foliations must be constructed to capture the full temperature dependence of entanglement entropy.

In order to systematically derive suitable finite temperature geometries, we must solve Einstein's equations in the bulk with a constant, negative cosmological constant, with our curved $2 d$ metric of interest given as a boundary condition. Finding solutions of the Einstein equation with a prescribed boundary metric is a well-studied problem. See for example, Ref. 38. Our strategy as presented above differs from the one in the aforementioned reference, but yields finite temperature results that agree very well with numerics. We will however, remark briefly on the more systematic approach, which involves solving Einstein's equations exactly for a given boundary metric. The advantage of our foliation-based approach, is that we need not find new geodesics in our bulk geometry; we can simply use the geodesics from the BTZ spacetime, being sure to implement an appropriate UV cutoff.

Finally, We will also look briefly at a larger class of deformations known as "solution-generating diffeomorphisms". In particular, we will discuss a holographic description of these inhomogeneous systems, and their entanglement properties.

\section{DIFFERENT FOLIATIONS IN ADS/CFT AND ENTANGLEMENT ENTROPY}

In this section, we collect necessary ingredients to describe the holographic duals of rainbow and SSD deformations, and the calculations of entanglement entropy.

\section{A. Different foliations in AdS/CFT}

In the AdS/CFT correspondence, the CFT is defined ("lives") on an asymptotic boundary of AdS. What concerns us in developing our holographic description of inhomogeneous systems is the fact that the AdS spacetime can be foliated (sliced) in various different ways. Different foliations (slicings) give rise to different asymptotic boundaries, and hence correspond to different situations on the CFT side. (See below, in particular Sec. II A 4.)

For example, consider the Poincaré patch of AdS described by the metric:

$$
d s_{A d S_{3}}^{2}=R^{2} \frac{d z^{2}+d x^{2}-d t^{2}}{z^{2}}
$$

where $z>0$ and $-\infty<t, x<+\infty$, and $R$ is the radius of AdS. (This coordinate patch covers only half of $A d S_{3}$.) In these coordinates, $A d S_{3}$ is foliated by $(1+1) \mathrm{d}$ flat spacetimes described by the coordinate $(t, x)$ (Fig. 1). Realized at the asymptotic boundary $z \rightarrow 0$ is the CFT in its ground state defined on the infinite line $-\infty<x<$ $+\infty$. Here, as usual, the central charge of the CFT is $c=3 R /\left(2 G_{N}\right)$ where $G_{N}$ is the Newton constant.

\section{Rindler evolution}

$A d S_{3}$ has foliations other than the one suggested by the Poincaré metric. Let us consider the Rindler-AdS foliation, ${ }^{39-42}$ which can be obtained from the Poincaré metric by the $z$-independent coordinate transformation,

$$
t=u \sinh \left(h t^{\prime}\right), \quad x=u \cosh \left(h t^{\prime}\right),
$$

where $u>0$ and $-\infty<t^{\prime}<\infty$. This coordinate covers half of the original space, so that the system is at a finite Unruh temperature. The metric in this coordinate is

$$
d s_{A d S_{3}}^{2}=\frac{R^{2}}{z^{2}}\left[\left(-u^{2} d t^{\prime 2}+d u^{2}\right)+d z^{2}\right] .
$$

It is also instructive to introduce a tortoise coordinate $x^{\prime} \in[-\infty,+\infty]$ by $u=: h^{-1} e^{h x^{\prime}}$. The metric is

$$
d s_{A d S_{3}}^{2}=\frac{R^{2}}{z^{2}}\left[e^{2 h x^{\prime}}\left(-d t^{\prime 2}+d x^{\prime 2}\right)+d z^{2}\right] .
$$

Here,

$$
d s_{\text {Rindler }}^{2}=e^{2 h x^{\prime}}\left(-d t^{\prime 2}+d x^{\prime 2}\right)
$$

is the line element of the $2 \mathrm{~d}$ Rindler space. From Eq. (14), we read off the Rindler Hamiltonian,

$$
H_{\text {Rindler }}=\int_{0}^{\infty} d u u \mathcal{H}(u) .
$$

This is nothing but the entanglement Hamiltonian of the half space, starting from the vacuum of CFT. (The entanglement Hamiltonian of the finite interval can be discussed similarly. ${ }^{27,43}$ )

In the Rindler foliation, the coordinate system (13) covers only the half of the boundary. In other words, observers in the coordinate patch (13) cannot access the other half. The observers are hence effectively at finite temperature. ${ }^{44}$ Correspondingly, in the bulk, there is a topological black hole. ${ }^{43}$ Other foliations of $A d S_{3}$ lead to other inhomogeneous field theories on the boundary.

\section{Rainbow chain}

To realize the rainbow chain, we foliate $A d S_{3}$ by $A d S_{2} \cdot{ }^{35-37}$ The corresponding metric can be obtained from the Poincaré metric (12) by the following $t$ independent coordinate transformation:

$$
z=\eta \cos (h \Theta), \quad x=\eta \sin (h \Theta),
$$

where $\eta>0$ and $-\pi / 2 h<\Theta<\pi / 2 h$. Contours of constant $\eta$ and $\Theta$ are plotted in Fig. 1. The metric is given by

$$
d s_{A d S_{3}}^{2}=\left[\frac{h^{2} R^{2}}{\cos ^{2}(h \Theta)}\right]\left[d \Theta^{2}+d s_{A d S_{2}}^{2}\right],
$$



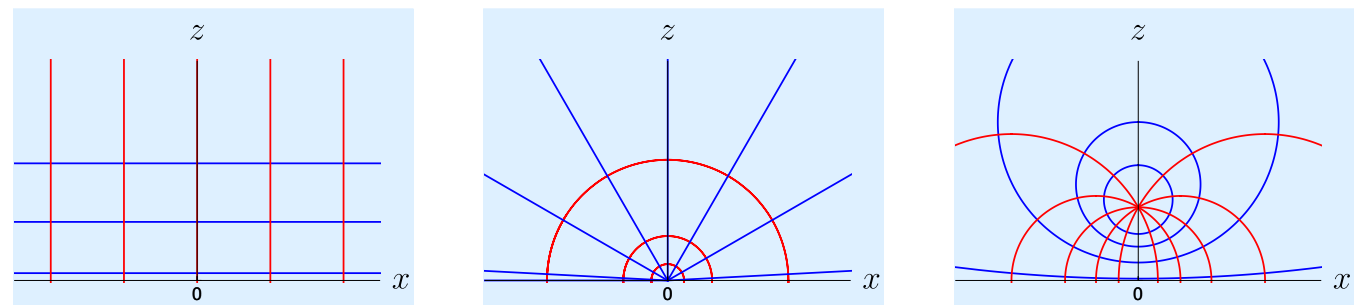

FIG. 1. Three different foliations of $A d S_{3}$ by flat Minkowski spaces (Left), $A d S_{2}$ (Middle), and 2d spaces with the metric (11) (Left). (Middle) Lines of constant $\eta$ (red) and $\Theta$ (blue) for the rainbow coordinate transformation (18); (Right) Lines of constant $v$ (red) and $u$ (blue) for the SSD coordinate transformation (21) with $a=1$. Both are plotted in the original Poincaré spatial coordinates, $x$ and $z$.

where $d s_{A d S_{2}}^{2}$ is given by (8). There are two asymptotic boundaries, one at $\Theta=+\pi / 2 h$ and the other at $\Theta=-\pi / 2 h$. There are two CFTs, one for each boundary, which are put on $A d S_{2}$. These two CFTs are not decoupled, but are connected at the boundary of $A d S_{2}$. The ground state is highly entangled between the two CFTs.

\section{Möbius and SSD evolution}

The prescription of generating the holographic dual of the rainbow chain can be generalized, by considering different $t$-independent coordinate transformations than (18). Let us now consider a coordinate map

$$
u+i v=\log (z+i x+a)-\log (z+i x-a),
$$

where $a$ is a real parameter. Inverting this and separating the real and imaginary parts, we have:

$$
z=\frac{a \sinh u}{\cosh u-\cos v}, \quad x=\frac{-a \sin v}{\cosh u-\cos v},
$$

where $u \in[0, \infty)$ and $v \in[0,2 \pi)$. This coordinate transformation is plotted in Fig. 1. Our Poincaré metric thus becomes

$$
d s_{A d S_{3}}^{2}=\frac{1}{\sinh ^{2} u}\left[d u^{2}+d v^{2}-a^{-2}(\cosh u-\cos v)^{2} d t^{2}\right]
$$

and our conformal boundary now occurs as $u \rightarrow 0$. Near the boundary $u \rightarrow u_{0}$, the boundary metric is given by

$$
d s^{2}=\left[\frac{1}{a \tanh u_{0}}\right]^{2}\left[-\left(1-\frac{\cos v}{\cosh u_{0}}\right)^{2} d t^{2}+\frac{a^{2} d v^{2}}{\cosh ^{2} u_{0}}\right]
$$

where $u_{0}$ is the UV cutoff. To make contact with (10), we introduce a parameter $\gamma$ by

$$
\tanh 2 \gamma=\frac{1}{\cosh u_{0}}
$$

By further introducing $L$ by

$$
\frac{L}{2 \pi}=\frac{a}{\cosh u_{0}} \stackrel{u_{0} \rightarrow 0}{\longrightarrow} a,
$$

and the change of variable $v=2 \pi x / L$, we arrive at

$$
d s^{2}=\left[\frac{\cosh 2 \gamma}{a}\right]^{2} d s_{\text {Mobius }}^{2},
$$

where $d s_{\text {Mobius }}^{2}$ is given by (11). In our metric (23), the UV cutoff $u_{0}$ plays the role of $\gamma$; one can then see that our foliation realizes a regularized version of the SSD.

a. Dipolar limit It is also interesting to take the limit $a \rightarrow 0$, while keeping $z / a$ and $x / a$ finite; this is the dipolar limit. We then consider the coordinate transformation:

$$
u+i v=\frac{a}{z+i x} .
$$

Separating into real and imaginary parts, we have,

$$
x=\frac{-a v}{u^{2}+v^{2}}, \quad z=\frac{a u}{u^{2}+v^{2}} .
$$

The metric is then given by

$$
d s_{A d S_{3}}^{2}=\frac{R^{2}}{u^{2}}\left[d v^{2}-a^{-2}\left(u^{2}+v^{2}\right)^{2} d t^{2}+d u^{2}\right],
$$

where $u \rightarrow 0$ corresponds to the conformal boundary. Near the boundary $u \sim u_{0}$, the metric for a given slice is

$$
d s^{2}=\frac{R^{2}}{u_{0}^{2}}\left[-a^{-2}\left(v^{2}+u_{0}^{2}\right)^{2} d t^{2}+d v^{2}\right] .
$$

\section{Foliations and UV cutoff}

For each of the different coordinate transformations we considered above, we have an associated "natural" foliation; For example, in (19), we have a family of surfaces with $A d S_{2}$ metric parameterized by $\Theta$. To properly define CFTs in the asymptotic boundaries, we further need to introduce a UV cut off. We do so by taking our cutoff surface, i.e., the surface where we define our CFTs, to be 
one of the slices located near (but away from) the boundary (boundaries). This is the UV cutoff which is "consistent" or "natural" for a given foliation. In terms of the Poincaré coordinate (12) that we started with, this means that our the cutoff is position-dependent ( $x$-dependent). Assuming our bulk foliation is dictated by a coordinate transformation, $z=z(u, v)$ and $x=x(u, v)$ (where $u$ and $v$ are our new radial and transverse coordinates, respectively), we replace the UV cutoffs with their curvilinear counterparts:

$$
\left.\epsilon \rightarrow z(u=\epsilon, v(x)) \simeq \frac{\partial z(u, v)}{\partial u}\right|_{\substack{u=0 \\ v=v(x)}} \epsilon .
$$

It is worth emphasizing that it is because of the cutoff that we realize a "different CFT" as mentioned, e.g., in Ref. 40. For example, the metric of the type (19) was previously used to discuss holographic duals of boundary CFTs (BCFTs) ${ }^{45,46}$; In the $A d S / B C F T$ correspondence, one realizes a BCFT on $A d S_{2}$ which has a boundary (or boundaries). There, however, one imposes the "original" cutoff using the flat Minkowski cutoff surfaces. ${ }^{45}$

\section{Solution generating diffeomorphisms (SGDs)}

We can construct a family of locally $A d S_{3}$ spacetimes with the appropriate asymptotic behavior (i.e. that preserve the form of the Fefferman-Graham metric, up to gauge transformations) by acting on the vacuum metric with a certain class of diffeomorphisms. ${ }^{42,47}$ Applying one of these transformations corresponds to exciting a state in the CFT. ${ }^{48-50}$ We say therefore that they are "solution-generating diffeomorphisms".

Using light cone coordinates for the boundary, $x_{ \pm}=$ $t \pm x$, we can parameterize the transformations as follows:

$$
x_{ \pm}=f_{ \pm}\left(\tilde{x}_{ \pm}\right), \quad z=\tilde{z} \sqrt{f_{+}^{\prime}\left(\tilde{x}_{+}\right) f_{-}^{\prime}\left(\tilde{x}_{-}\right)} .
$$

Starting from the Poincaré metric (12), we obtain the following bulk metric in the new coordinates:

$$
\begin{aligned}
d s_{A d S_{3}}^{2}= & \frac{d \tilde{z}^{2}-d \tilde{x}_{+} d \tilde{x}_{-}}{\tilde{z}^{2}}+\left(A_{+} d \tilde{x}_{+}+A_{-} d \tilde{x}_{-}\right)^{2} \\
& +\frac{2 d \tilde{z}}{\tilde{z}}\left(A_{+} d \tilde{x}_{+}+A_{-} d \tilde{x}_{-}\right)
\end{aligned}
$$

where $A_{ \pm}=-(1 / 2) f_{ \pm}^{\prime \prime}\left(\tilde{x}_{ \pm}\right) / f_{ \pm}^{\prime}\left(\tilde{x}_{ \pm}\right)$.

Although these diffeomorphisms preserve the form of the metric and are therefore trivial gauge transformations from the perspective of the bulk, they are nontrivial at the asymptotic boundary. This nontriviality can be understood by observing that the SGDs result in nonzero contributions to the boundary stress tensor from the Schwarzian derivative:

$$
\begin{aligned}
T_{ \pm \pm} & =\frac{c}{48 \pi f_{ \pm}^{\prime}\left(\tilde{x}_{ \pm}\right)^{2}}\left[3 f_{ \pm}^{\prime \prime}\left(\tilde{x}_{ \pm}\right)^{2}-2 f_{ \pm}^{\prime}\left(\tilde{x}_{ \pm}\right) f_{ \pm}^{\prime \prime \prime}\left(\tilde{x}_{ \pm}\right)\right] \\
T_{+-} & =0
\end{aligned}
$$

With the stress-tensor in hand, we can write the metric (33) in Fefferman-Graham form:

$$
\begin{aligned}
d s_{A d S_{3}}^{2} & =\frac{d z^{2}}{z^{2}}-\frac{1}{z^{2}}\left[d x_{+} d x_{-}\right. \\
& +\frac{z^{2}}{4}\left(L\left(x_{+}\right) d x_{+}^{2}+\bar{L}\left(x_{-}\right) d x_{-}^{2}\right) \\
& \left.+\frac{z^{4}}{16} L\left(x_{+}\right) \bar{L}\left(x_{-}\right) d x_{+} d x_{-}\right]
\end{aligned}
$$

where $L\left(x_{+}\right)=\frac{48 \pi}{c} T_{++}$and $\bar{L}\left(x_{-}\right)=\frac{48 \pi}{c} T_{--}{ }^{38}$. The nonzero energy-momentum tensor means that we are in an excited state of the original CFT. Indeed, we can write this state explicitly by finding a unitary representation of our diffeomorphisms. For a diffeomorphism (written in complex coordinates $z=x+i t) f(z)=\sum_{n=-\infty}^{\infty} \epsilon_{n} z^{-n+1}$, this state is given by $|\Omega\rangle_{f}=U(\epsilon)|0\rangle$, where wh $^{50} U(\epsilon)=$ $\exp \left(\sum_{n=-\infty}^{\infty} \epsilon_{n} L_{-n}\right)$, where, $L_{-n}$ are the standard Virasoro generators.

Note that $z$ and $x_{ \pm}$in (37) are not the same as the original Poincaré coordinates; they are chosen specifically to put the metric in Fefferman-Graham form. In this gauge, the metric has a horizon located at $z=z_{H}=$ $2\left(L\left(x_{+}\right) \bar{L}\left(x_{-}\right)\right)^{-1 / 4}$. As $z \rightarrow 0$, we see that we recover the flat Minkowski metric. To investigate our excited state, we must use a constant $\tilde{z}=\epsilon$ cutoff corresponding to the curvilinear cutoff

$$
z=\epsilon \sqrt{f_{+}^{\prime}\left(\tilde{x}_{+}\right) f_{-}^{\prime}\left(\tilde{x}_{-}\right)}
$$

The essential physics in both the SGD protocol and the prescription we have outlined in this paper is the same. In both cases, we pick a UV cutoff for our bulk spacetime that depends on the transverse Poincaré coordinates. In our prescription, we arrive at the curvilinear cutoff by performing a particular time-independent coordinate transformation, while in the SGD case, the cutoff emerges as a natural result of extending a conformal transformation into the bulk. A key difference between the two is that SGDs are in general time-dependent, since they are formulated in terms of light-cone coordinates. Indeed, the class of Weyl transformations that the SGDs induce on the UV cutoff surface is limited to those of the form $\exp \left(2 \phi\left(\tilde{x}_{+}, \tilde{x}_{-}\right)\right)=f_{+}^{\prime}\left(\tilde{x}_{+}\right) f_{-}^{\prime}\left(\tilde{x}_{-}\right)$, while in our prescription, the Weyl transformations depend purely on the transverse spatial coordinate (e.g. $\exp (2 \phi(u))=1 / u^{2}$ for the rainbow chain). Nevertheless, SGDs can be used to construct many interesting foliations of $A d S_{3}$. Examples include the Rindler foliation mentioned previously, the Hopf fibration ${ }^{51}$, and various two-sided geometries mentioned in Ref. 49. 


\section{B. Entanglement entropy}

\section{Zero temperature}

Once we have obtained a foliation of $A d S_{3}$ corresponding to our inhomogeneous system of interest, we can use the Ryu-Takayanagi procedure to compute the bipartite entanglement entropy of a particular interval on the boundary. ${ }^{52-54}$ We start by using the known result for the zero temperature holographic entanglement entropy for an interval $\left[x_{1}, x_{2}\right]$ on the asymptotic boundary of the Poincaré patch:

$$
S_{A}^{\text {holo }}\left(x_{1}, x_{2}\right)=\frac{c}{3} \log \left[\frac{x_{2}-x_{1}}{\sqrt{\epsilon_{1}} \sqrt{\epsilon_{2}}}\right] .
$$

As before, assuming our bulk foliation is dictated by a coordinate transformation, $z=z(u, v)$ and $x=x(u, v)$ (where $u$ and $v$ are our new radial and transverse coordinates, respectively), we replace the UV cutoffs, $\epsilon_{1}$ and $\epsilon_{2}$ with their curvilinear counterparts:

$$
\epsilon_{i} \rightarrow z\left(u=\epsilon, v\left(x_{i}\right)\right)=\left.\frac{\partial z(u, v)}{\partial u}\right|_{\substack{u=0 \\ v_{i}=v\left(x_{i}\right)}} \epsilon
$$

Equation (39) then becomes

$$
S_{A}^{\text {holo }}\left(v_{1}, v_{2}\right)=\frac{c}{3} \log \left[\frac{x\left(u=0, v_{2}\right)-x\left(u=0, v_{1}\right)}{\epsilon \sqrt{\frac{\partial z\left(u=0, v_{1}\right)}{\partial u} \frac{\partial z\left(u=0, v_{2}\right)}{\partial u}}}\right] .
$$

For example, in the dipole foliation, where $z=\frac{a u}{u^{2}+v^{2}}$, the UV-cutoff and transverse coordinate transform as

$$
\epsilon_{i}=\frac{a}{v_{i}^{2}} \epsilon, \quad x_{i}=x\left(u=0, v_{i}\right)=\frac{-a}{v_{i}} .
$$

We can plug these into (39) to find the holographic entropy of the dipole foliation. Similarly, for metric (33), using the cutoff (38), we can compute the entanglement entropy of an interval in the $\tilde{x}_{ \pm}$coordinates: ${ }^{48,55}$

$$
\begin{aligned}
& S_{A}\left(x_{1}, x_{2}\right) \\
& =\frac{c}{12} \log \left[\frac{\mathcal{L}\left(\tilde{x}_{1+}, \tilde{x}_{2+}\right)^{2} \mathcal{L}\left(\tilde{x}_{1-}, \tilde{x}_{2-}\right)^{2}}{\epsilon^{4} f_{+}^{\prime}\left(\tilde{x}_{1+}\right) f_{+}^{\prime}\left(\tilde{x}_{2+}\right) f_{-}^{\prime}\left(\tilde{x}_{1-}\right) f_{-}^{\prime}\left(\tilde{x}_{2-}\right)}\right],
\end{aligned}
$$

where $\mathcal{L}\left(\tilde{x}_{1 \pm}, \tilde{x}_{2 \pm}\right)$ is the proper length on the boundary between $\tilde{x}_{1 \pm}$ and $\tilde{x}_{2 \pm}$.

\section{Finite temperature}

Computing holographic entanglement entropy for inhomogeneous systems at finite temperature is a less trivial matter than the zero temperature case; we need to find a proper foliation of the BTZ black hole solution with a given metric on the boundary. While this procedure can be implemented in certain cases (as described below), we will use a different approach in the bulk of the paper, in which we cut off the BTZ black hole spacetime with the same curvilinear UV cutoff used in the previous section. Although approximate, this approach allows us to use known results for geodesic lengths of entangling surfaces in the BTZ spacetime. This will allow us to avoid solving for potentially complicated geodesics that the bulk metrics from the exact treatment would yield.

Let us first outline the exact approach following the results in Ref. 38. We begin by specifying an arbitrary static $(1+1) \mathrm{d}$ boundary metric on the $x-t$ plane:

$$
d s_{\partial}^{2}=-h(x) d t^{2}+\frac{d x^{2}}{h(x)} .
$$

Note that any $(1+1) d$ static metric can be written this way, up to a spatial coordinate transformation. We can solve the resulting Dirichlet problem in the FeffermanGraham gauge in terms of two undetermined constants, $J$ and $B$. Assuming $J=0$ (to avoid a cross term in the metric), the resulting bulk metric is

$$
\begin{aligned}
d s^{2}= & \frac{R^{2} d z^{2}}{z^{2}}+\frac{1}{z^{2}}\left[-h\left(1+\frac{R^{2}}{16} \frac{h^{\prime 2}-B^{2}}{h} z^{2}\right)^{2} d t^{2}\right. \\
& \left.+\frac{1}{h}\left(1+\frac{R^{2}}{4} h^{\prime \prime} z^{2}-\frac{R^{2}}{16} \frac{h^{\prime 2}-B^{2}}{h} z^{2}\right)^{2} d x^{2}\right]
\end{aligned}
$$

For $h(x)=1$, the above reduces to the FeffermanGraham form of the BTZ metric ${ }^{56}$ with a horizon at $z_{H}=4 / R B$. The parameter $B$ thus corresponds to temperature in the boundary CFT.

For a more general $h(x)$, a more interesting horizon will be present. For example, if we start with the $A d S_{2}$ boundary metric (8) making the coordinate change $x=$ $1 / \eta$ puts the metric in the form of (44) with $h(x)=x^{2}$ (here we set the curvature scale $h$ in (8) to be 1 for simplicity). Plugging this into (45) and transforming back to the original $\eta$ coordinate gives us the following bulk solution:

$$
\begin{aligned}
d s^{2}=\frac{d z^{2}}{z^{2}}+\frac{1}{z^{2} \eta^{2}} & {\left[\left(1+R^{2}\left(1+B^{2} \eta^{2}\right) z^{2}\right)^{2} d x^{2}\right.} \\
- & \left.\left(1+R^{2}\left(1-B^{2} \eta^{2}\right) z^{2}\right)^{2} d t^{2}\right]
\end{aligned}
$$

which appears to have an interesting horizon at $z=$ $1 /\left(R \sqrt{B^{2} \eta^{2}-1}\right)$. Note that as $z \rightarrow 0$, we see our bulk metric reduce to the $A d S_{2}$ foliation that we expect from the rainbow chain dual.

Computing the entanglement entropy would entail picking two boundary points, $\eta_{1}$ and $\eta_{2}$, on a fixed time slice of (46), and computing the length of the spacelike geodesic between them. This would of course require a UV cutoff, which we would choose to be a constant $z=\epsilon$. Solving the Euler-Lagrange equations for the spacelike geodesics of (46) is nontrivial, and will be different for each inhomogeneous system of interest. It is for this reason that we use the approximate approach in this paper. 
This approximation for finding the finite temperature entanglement entropy in inhomogeneous systems requires two inputs. The first is the well-known result for the finite temperature holographic entanglement entropy ${ }^{54}$, which in Poincaré coordinates takes the form

$S_{A}^{\text {holo }}\left(x_{1}, x_{2} ; \beta\right)=\frac{c}{3} \log \left[\frac{\beta}{\pi \sqrt{\epsilon_{1}} \sqrt{\epsilon_{2}}} \sinh \left(\frac{\pi\left(x_{2}-x_{1}\right)}{\beta}\right)\right]$.

For reference, in Poincaré coordinates the BTZ black hole metric is

$$
\begin{aligned}
d s_{B T Z}^{2} & =R^{2}\left[-\frac{f(z) d t^{2}}{z^{2}}+\frac{d z^{2}}{f(z) z^{2}}+\frac{d x^{2}}{z^{2}}\right] \\
f(z) & =1-\frac{z^{2}}{z_{H}^{2}}
\end{aligned}
$$

where $z_{H}$ is related to the inverse temperature $\beta$ at the boundary via $\beta=2 \pi z_{H}$.

With (47) in hand, we can add the second ingredient, and replace $\epsilon_{1}$ and $\epsilon_{2}$ with appropriate curvilinear cutoffs. As in the previous subsection, we can find these cutoffs from the bulk coordinate change that produced our zero temperature foliation. Combining (40) and the coordinate transformations on the boundary with (47) yields a result for the finite temperature bipartite entanglement entropy in an inhomogeneous CFT:

$$
\begin{aligned}
& S_{A}^{\text {holo }}\left(v_{1}, v_{2} ; \beta\right) \\
& =\frac{c}{3} \log \left[\frac{\beta}{\pi \epsilon} \frac{\sinh \left(\frac{\pi}{\beta}\left(x\left(u=0, v_{2}\right)-x\left(u=0, v_{1}\right)\right)\right)}{\sqrt{\frac{\partial z\left(u=0, v_{1}\right)}{\partial u} \frac{\partial z\left(u=0, v_{2}\right)}{\partial u}}}\right] .
\end{aligned}
$$

The above can be considered our finite temperature master formula for entanglement entropy, and we will use it throughout the paper.

Though this approach is quite simple, it has one primary limitation. The foliations that we use in vacuum $A d S_{3}$ are not always well-suited for the black hole spacetime. Indeed, the curved cutoffs we use in the BTZ spacetime will often collide with the black hole horizon. For example, the dipole foliation uses a cutoff at $\epsilon_{z}=a \epsilon / v^{2}$. For a BTZ spacetime with a horizon at $z=z_{H}$, this cutoff surface will actually be inside of the black hole horizon for $v<\sqrt{a \epsilon / z_{H}}$. This means that a large part of our boundary system could be inaccessible at very high temperatures (very small $z_{H}$ ). This issue will become apparent in the ensuing comparisons between numerics and holographic results, which will diverge from each other for sufficiently large temperatures and in for intervals in certain regions of the boundary. However, for most of the boundary theory, and for a wide range of temperatures, the approximation appears to yield results that agree quite well with numerics. We will thus use it in the remainder of this paper.

\section{CFT on curved spacetime}

The connection between the entanglement scaling of inhomogeneous and homogeneous CFTs can be also seen without using holography. Recall that the entanglement entropy for a single interval is given by the 2-point function of twist operators located at two points $u_{1}$ and $u_{2}$ at a fixed time. Let us consider the correlators of arbitrary operators, $O_{i}\left(x_{i}\right)$, of CFT put on the flat Euclidean metric $d s_{0}(x)^{2}=d x^{2}+d \tau^{2}$, and the correlators of the same CFT put on the curved metric

$$
\begin{aligned}
d s^{2} & =e^{2 \sigma(x)} d s_{0}^{2}=e^{2 \sigma(x)} d \bar{z} d z \\
& =e^{2 \sigma(x(u))}\left[\left(\frac{d x}{d u}\right)^{2} d u^{2}+d \tau^{2}\right]
\end{aligned}
$$

where $z=x+i \tau$ and $u$ is a spatial coordinate appropriate for the curved metric. CFT gives us the following relationship between correlation functions under Weyl and coordinate transformations:

$$
\begin{aligned}
& \left\langle O_{1}\left(u_{1}\right) O_{2}\left(u_{2}\right) \cdots\right\rangle_{d s^{2}} \\
& =e^{-\sum_{i} \Delta_{i} \sigma\left(u_{i}\left(x_{i}\right)\right)}\left\langle O_{1}\left(x_{1}\right) O_{2}\left(x_{2}\right) \cdots\right\rangle_{d s_{0}(x)^{2}} \\
& =e^{-\sum_{i} \Delta_{i} \sigma\left(u_{i}\right)}\left\langle O_{1}\left(u_{1}\right) O_{2}\left(u_{2}\right) \cdots\right\rangle_{d s_{0}(u)^{2}}
\end{aligned}
$$

where $\Delta_{i}$ are the scaling dimensions of the operators, which for the $n$-fold twist operators are

$$
\Delta^{(n)}=\frac{c}{12}\left(n-\frac{1}{n}\right) .
$$

Since $d s_{0}(x)^{2}$ is just the Euclidean metric, we can write down the known result for the two-point function of the twist operators at a fixed time (assuming we are on the infinite complex plane):

$$
\begin{aligned}
\left.\operatorname{Tr}\left(\rho_{A}^{n}\right)\right|_{d s_{0}^{2}} & =\left\langle\sigma_{n}\left(x_{1}, \tau=0\right) \sigma_{-n}\left(x_{2}, \tau=0\right)\right\rangle_{d s_{0}^{2}} \\
& =c_{n}\left[\frac{x_{1}-x_{2}}{\epsilon}\right]^{-2 \Delta^{(n)}}
\end{aligned}
$$

where $c_{n}$ is a constant coefficient that will make an $\mathcal{O}(1)$ contribution to the entanglement entropy. The (Renyi) entanglement entropy in the curved background follows from this expression by multiplying the Weyl factor and transforming back to the original coordinates.

\section{HOLOGRAPHIC DUAL OF RAINBOW CHAIN}

We now discuss the holographic dual of the rainbow chain, (19), in some detail. In particular, we study the entanglement entropy for a given connected region $A$ of the boundary. As the system is inhomogeneous, not only the size of the subregion, but also its location matters. Here, we mainly consider two situations: 
- "Defect entanglement": where we consider an entangling cut that separates the origin ("defect") from the rest of the system.

- "Half chain entanglement": where we consider an entangling cut that emanates from the origin (defect). This entangling cut thus splits the original system into two halves.

In addition, both of these two situations can be studied at finite temperature.

\section{A. Defect entanglement at zero temperature}

Let us first discuss the defect entanglement. Recall the metric (19) for the $A d S$ foliation. We choose, in the Poincare coordinates, $x \in\left[-\eta_{0},+\eta_{0}\right]$ on the boundary as the region of our interest. The geodesic $\Gamma$ anchored at $(\eta, \Theta)=\left(\eta_{0}, \pm \pi / 2 h\right)$ is a semi-circle on the $z$ - $x$ plane, i.e., $\eta(\Theta)=$ const. $=\eta_{0}$. The length of $\Gamma, \operatorname{Len}(\Gamma)=\int_{\Gamma} d s$, is given by

$$
\operatorname{Len}(\Gamma)=h R \int_{-\pi / 2 h}^{\pi / 2 h} \frac{d \Theta}{\cos (h \Theta)} \sqrt{1+\frac{1}{h^{2} \eta^{2}}\left(\frac{d \eta}{d \Theta}\right)^{2}} .
$$

This integral is divergent, and one has to introduce a cutoff, $\pm \pi / 2 h \rightarrow \pm(\pi / 2 h-\epsilon)$, where $\epsilon$ is an $\eta$-independent constant. The regularized length is a constant independent of $\eta_{0}$ (i.e., independent of the size of the subsystem):

$$
S_{A}\left(\eta_{0}\right)=\frac{c}{3} \log \left(\frac{2}{\epsilon h}\right)+\mathcal{O}(1)
$$

This agrees with the known behavior of the defect entanglement entropy in the rainbow chain. It does, however, depend on $1 / h$, the length scale introduced by the rainbow defect (i.e., the $A d S_{2}$ radius). One can check that this is the same result we would have obtained using (39).

The "defect entanglement" (55) we have just encountered resembles the constant "boundary entanglement" found in a BCFT. The latter, however, is an $\mathcal{O}(1)$ constant correction to the logarithmically divergent leading order term, whereas the defect entanglement is constant, but $\mathcal{O}(\log (\epsilon))$. Indeed, in $A d S / B C F T$, a zero tension brane anchored at a boundary point of $A d S_{3}$ is dual to a half-space BCFT on the conformal boundary of the spacetime. ${ }^{46}$ This particular $A d S / B C F T$ setup strongly resembles our effective holographic description of the rainbow chain, and indeed, $A d S_{2}$ foliation of the bulk captures the breakdown of the global symmetry group from $S O(2,2)$ to $S O(2,1)$. However, a zero tension brane (which is just an artifact of a coordinate transformation) yields zero boundary entropy, and the defect entanglement simply reflects the contributions of the ends of our entanglement interval to the entropy. A boundary entropy would reflect the entropy due to the presence of a tensionful brane. Adding such a brane could prove to be an interesting extension of the rainbow chain.

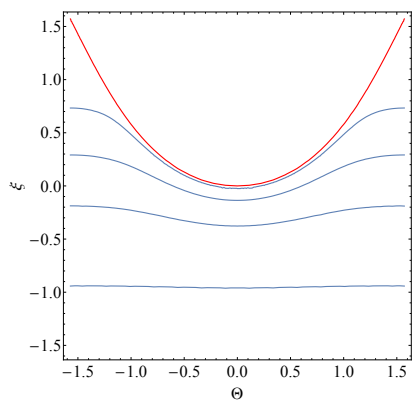

FIG. 2. The black hole horizon (red) and several defectcrossing geodesics (blue) plotted in $\xi$ and $\Theta$ coordinates where $d \xi / \cos \xi=d \eta / \eta$ (see Eq. (62)).

\section{B. Defect entanglement at finite temperature}

We can bring this metric back to the one we want to work with, Eq. (18). For reference, the geodesic in these coordinates takes the form

$$
\sqrt{1-\frac{\eta^{2} \cos ^{2}(h \Theta)}{z_{H}^{2}}}=\sqrt{1-\frac{z_{*}^{2}}{z_{H}^{2}}} \cosh \left[\frac{\eta \sin (h \Theta)}{z_{H}}\right] .
$$

In Fig. 2, we have plotted the geodesic for several values of $z_{*}$ in blue, with the black hole horizon plotted in red.

We will cut our spatial slice off at a constant $\Theta_{\epsilon}=$ $\pi / 2 h-\epsilon$. Expressed as a cutoff in the $z$ coordinate, the cutoff now depends on $x_{0}=\eta_{0}$ as follows:

$$
\epsilon_{z}=\eta_{0} \cos \left(h \Theta_{\epsilon}\right) \approx \eta_{0} h \epsilon .
$$

Thus, using our master formula, (49), our defect entanglement entropy reduces to

$$
S_{A}\left(\eta_{0} ; \beta\right)=\frac{c}{3} \log \left[\frac{\beta}{\pi \epsilon h \eta_{0}} \sinh \left(\frac{2 \pi \eta_{0}}{\beta}\right)\right] .
$$

Taking the zero temperature limit, $\beta \rightarrow \infty$, we recover (55). The proper length of the boundary interval

$$
\ell=\frac{2}{h} \int_{\epsilon}^{\eta_{0}} \frac{d \eta}{\eta}
$$

In terms of the proper length, the defect entanglement entropy is :

$$
S_{A}(x ; \beta, \epsilon)=\frac{c}{3} \log \left[\frac{\beta}{\pi h e^{h \ell}} \sinh \left(\frac{2 \pi \epsilon e^{h \ell}}{\beta}\right)\right]+\cdots,
$$

where $\cdots$ is a non-universal part depending on the UV cutoff.

a. Comparison with numerics To verify the finite temperature entanglement results for the rainbow chain, we can numerically compute the entanglement entropy of an interval of space at a given temperature for the Hamiltonian (4). All numerical computations were done with open boundary conditions. In Fig. 3 we have plotted the numerically-computed entanglement entropy for 


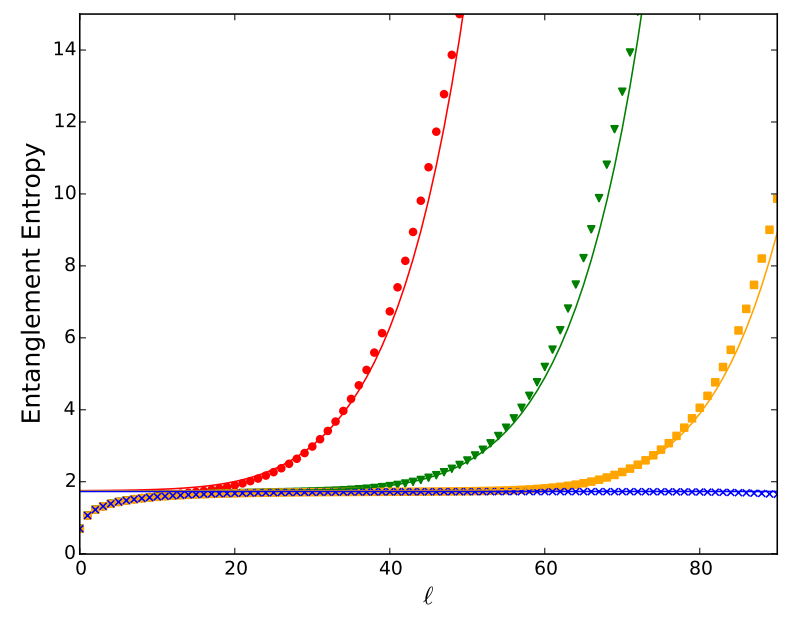

FIG. 3. The numerically computed "defect" entanglement entropy as a function of the proper length of the interval about the defect at $h=0.1$ and $\epsilon=0.5$ and at finite temperatures $\beta=100,1000,10000, \infty$ from the top. The numerical data are fitted to (60), where we treat the non-universal constant part (independent of $\ell$ and $\beta$ ) as a fitting parameter.

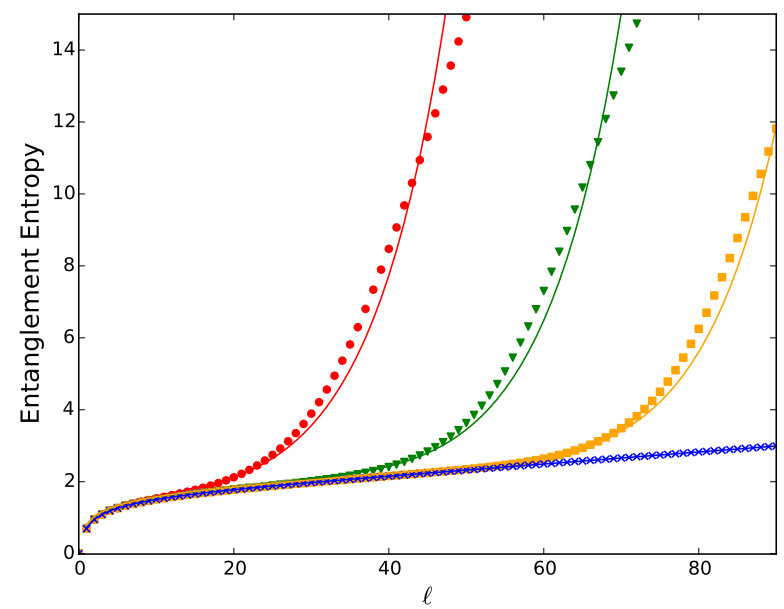

FIG. 4. The numerically computed "half-chain" entanglement entropy at finite temperature at $h=0.1$ for $\beta=$ $100,1000,10000, \infty$ from the top. The numerical data is fitted to Eq. (65), where we treat the non-universal constant part (independent of $\ell$ and $\beta$ ) as a fitting parameter.

an interval symmetric about the defect at the origin of the chain. We can confirm the rapid asymptote at zero temperature of this entropy to a constant, independent of the length of the interval, but dependent upon $h$, the rainbow curvature scale. Also plotted in Fig. 3 is the defect entanglement for several different temperatures, fitted with the analytic result (60).

\section{Half-chain entanglement at zero temperature}

Let us now discuss the half-chain entanglement. For this purpose, it is convenient to use the global coordinate:

$$
d s_{A d S_{3}}^{2}=\frac{h^{2} R^{2}}{\cos ^{2}(h \Theta)}\left(d \Theta^{2}+d s_{A d S_{2}}^{2}\right),
$$

where $d s_{A d S_{2}}^{2}$ is the metric of $A d S_{2}$ which will be described below. There are two asymptotic boundaries located at $\Theta= \pm \pi / 2 h$, where two CFTs, one for each boundary, are defined. As for $d s_{A d S_{2}}^{2}$, it is also given in terms of the global coordinate

$$
d s_{A d S_{2}}^{2}=\frac{1}{h^{2} \cos ^{2} \xi}\left(-d \tau^{2}+d \xi^{2}\right),
$$

$\tau \in(-\infty,+\infty)$ and $\xi \in(-\pi / 2, \pi / 2)$. There are two asymptotic boundaries at $\xi= \pm \pi / 2$. The two CFTs are connected at the boundaries of $A d S_{2}$.

Combining the coordinate transformation (18) with (39), we obtain the half-chain entanglement entropy. We can express this in terms of the proper length via $\ell=\frac{1}{h} \int_{\eta_{1}}^{\eta_{2}} \frac{d \eta}{\eta} \rightarrow \eta_{2}=\eta_{1} \exp (h \ell)$ :

$$
S_{A}(\ell)=\frac{c}{3} \log \left[\frac{2}{\epsilon h} \sinh \left(\frac{h \ell}{2}\right)\right] .
$$

The most striking feature about this zero temperature result is that it demonstrates a volume law growth for larger values of $\ell$, with $h$ acting as an effective temperature. This is consistent with results from previous work on the rainbow chain, ${ }^{3,4}$ where it was found that the rainbow phase strongly resembles a thermofield double state.

\section{Half-chain entanglement at finite temperature}

We can apply the same procedure of cutting off the geodesics in the BTZ spacetime at $\epsilon_{z}=\eta_{1,2} h \epsilon$. Here, we consider a geodesic anchored at arbitrary $\Theta=\pi / 2 h$ boundary points $\eta_{1}$ and $\eta_{2}$, where $\eta_{2}>\eta_{1}$. Combining these cutoffs with our finite temperature master formula (49) yields

$$
S_{A}\left(\eta_{1}, \eta_{2} ; \beta\right)=\frac{c}{3} \log \left[\frac{\beta}{\epsilon \pi h \sqrt{\eta_{1} \eta_{2}}} \sinh \left(\frac{\pi\left(\eta_{2}-\eta_{1}\right)}{\beta}\right)\right] .
$$

In terms of the proper length $\ell$, the half-chain entanglement is

$$
\begin{aligned}
& S_{A}\left(\ell ; \beta, \eta_{1}\right) \\
& =\frac{c}{3} \log \left[\frac{\beta}{\epsilon \pi h \eta_{1} e^{h \ell / 2}} \sinh \left(\frac{\pi \eta_{1}\left(e^{h \ell}-1\right)}{\beta}\right)\right] .
\end{aligned}
$$

The low temperature $\beta \rightarrow \infty$ limit agrees with (63).

We have also computed numerical results for the halfchain entanglement in Fig. 4, using the lattice model (4) 
with the hopping amplitudes (5). There, we choose two points $\eta_{1}$ and $\eta_{2}$, both located to the right of the defect, and set $\eta_{1}=12$ and increase $\ell$ by changing $\eta_{2}$. We use $\ell$ rather than the coordinates (7) We can see that the agreement between the numerical and holographic results is excellent at low temperatures.

\section{HOLOGRAPHIC DUAL OF SSD}

\section{A. Zero temperature}

Now let us compute the holographic entanglement entropy of the SSD at zero temperature using the metric (22). Let us first consider an interval $\left[v_{1}, v_{2}\right]$ (where $\left.0 \leq v_{1}, v_{2} \leq 2 \pi\right)$. Cutting off at $u=\epsilon$ corresponds to a $v$-dependent $z$ cutoff of $\epsilon_{z}=\frac{a \epsilon}{1-\cos (v)}$.

Using (39) and the above cutoff, we find that the zero temperature entanglement entropy is

$$
S_{A}\left(v_{1}, v_{2}\right)=\frac{c}{3} \log \left[\frac{2}{\epsilon} \sin \left(\frac{1}{2}\left|v_{2}-v_{1}\right|\right)\right] .
$$

The result (66) is just the entanglement entropy for a CFT on a finite length space with periodic boundary conditions and is what we expect from the SSD model. ${ }^{8}$

The above holographic results can be readily reproduced by a CFT calculation. The metric on our conformal boundary $(u=0)$ in imaginary time, $\tau$, is

$$
d s^{2}=d v^{2}+\frac{4}{a^{2}} \sin ^{4}\left(\frac{v}{2}\right) d \tau^{2} .
$$

We factor out the $\frac{4}{a^{2}} \sin ^{4}\left(\frac{v}{2}\right)$ and define a new variable

$$
d x=\frac{a d v}{2 \sin ^{2}\left(\frac{v}{2}\right)} \longrightarrow x=-a \cot \left(\frac{v}{2}\right)
$$

to write our metric as

$$
d s^{2}=e^{2 \sigma} d s_{0}^{2}, \quad e^{\sigma}=\frac{2}{a} \sin ^{2}\left(\frac{v}{2}\right)=\frac{2 a}{a^{2}+x^{2}},
$$

where $d s_{0}^{2}=d x^{2}+d \tau^{2}$. Since $x \in(-\infty, \infty)$, we can use the twist 2-point function on the full complex plane to compute the entanglement entropy (53). Then,

$$
\begin{aligned}
& \left.\operatorname{Tr}\left(\rho_{A}^{n}\right)\right|_{d s^{2}} \\
& =c_{n} e^{-\Delta^{(n)}\left[\sigma\left(u_{1}\right)+\sigma\left(u_{2}\right)\right]}\left[\frac{a \cot \left(\frac{v_{2}}{2}\right)-a \cot \left(\frac{v_{1}}{2}\right)}{\epsilon}\right]^{-2 \Delta^{(n)}} \\
& =c_{n}\left[2 \sin \left(\frac{1}{2}\left(v_{2}-v_{1}\right)\right)\right]^{-2 \Delta^{(n)}} .
\end{aligned}
$$

Taking the $\log$ and taking $n \rightarrow 1$, we reproduce the holographic result (66).

\section{B. Finite temperature}

We can once again use the cutoff $\epsilon_{z}=\frac{a \epsilon}{1-\cos (v)}$. This time we will plug it into (49) in order to obtain finite temperature results for the SSD. For the interval $\left[v_{1}, v_{2}\right]$, we obtain the following entanglement law:

$$
\begin{aligned}
& S_{A}\left(v_{1}, v_{2} ; \beta\right)=\frac{c}{3} \log \left[\frac{2}{a} \sin \left(\frac{v_{1}}{2}\right) \sin \left(\frac{v_{2}}{2}\right)\right] \\
&+\frac{c}{3} \log \left[\frac{\beta}{\pi \epsilon} \sinh \left(\frac{\pi a}{\beta} \frac{\sin \left(\frac{1}{2}\left(v_{2}-v_{1}\right)\right)}{\sin \left(\frac{v_{1}}{2}\right) \sin \left(\frac{v_{2}}{2}\right)}\right)\right] .
\end{aligned}
$$

We can confirm that at zero temperature, $S_{A}\left(v_{1}, v_{2} ; \beta \rightarrow\right.$ $\infty)$ agrees with the result from the previous section (66). Note, unlike the zero temperature result (66), (71) depends on $a$. If we center the interval about $\pi$, so that $v_{1}=\pi-v_{0}$ and $v_{2}=\pi+v_{0}\left(\right.$ where $\left.v_{0} \in[0, \pi)\right)$,

$$
\begin{aligned}
& S_{A}\left(\pi-v_{0}, \pi+v_{0} ; \beta\right) \\
& =\frac{c}{3} \log \left[\frac{2 \beta}{\pi a \epsilon} \cos ^{2}\left(\frac{v_{0}}{2}\right) \sinh \left(\frac{2 \pi a}{\beta} \tan \left(\frac{v_{0}}{2}\right)\right)\right] .
\end{aligned}
$$

For comparison with the SSD model put on a line of finite length $L$, we rescale $v_{i} \rightarrow x_{i}=L v_{i} / 2 \pi$ and recall that the parameter $a$ and $L$ are related by (25), $a=$ $L / 2 \pi$. The entanglement entropy is then given by

$$
\begin{aligned}
& S_{A}\left(x_{1}, x_{2} ; \beta\right)=\frac{c}{3} \log \left[\frac{4 \pi}{L} \sin \left(\frac{x_{1}}{\pi}\right) \sin \left(\frac{x_{2}}{\pi}\right)\right] \\
& +\frac{c}{3} \log \left[\frac{\beta}{\pi \epsilon} \sinh \left(\frac{L}{2 \beta} \frac{\sin \left(\frac{1}{\pi}\left(x_{2}-x_{1}\right)\right)}{\sin \left(\frac{x_{1}}{\pi}\right) \sin \left(\frac{x_{2}}{\pi}\right)}\right)\right] .
\end{aligned}
$$

Once again, if we center the interval about $\pi$, so that $v_{1}=\pi-v_{0}$ and $v_{2}=\pi+v_{0}\left(\right.$ where $v_{0} \in[0, \pi)$,

$$
\begin{aligned}
& S_{A}\left(\pi-v_{0}, \pi+v_{0} ; \beta\right) \\
& =\frac{c}{3} \log \left[\frac{4 \beta}{L \epsilon} \cos ^{2}\left(\frac{v_{0}}{2}\right) \sinh \left(\frac{L}{\beta} \tan \left(\frac{v_{0}}{2}\right)\right)\right] .
\end{aligned}
$$

In Fig. 5 we have plotted the entanglement entropy for several values of $\beta$, computed numerically from the Hamiltonian (4) with

$$
f_{j}=\frac{2}{a} \sin ^{2}\left(\frac{j \pi}{N}\right)=\frac{4 \pi}{N-1} \sin ^{2}\left(\frac{j \pi}{N}\right) .
$$

Here, we put the over all factor $2 / a=4 \pi /(N-1)$ since the boundary metric of our holographic setup is not $d s_{\text {Mobius }}^{2}$ itself, but $d s_{\text {Mobius }}^{2}$ multiplied by the factor $(\cosh 2 \gamma / a)^{2} \rightarrow(2 / a)^{2}$; See Eq. (11). The $N$-dependent multiplicative factor serves to compress the density of states of the SSD model. This quantity only becomes relevant to the entanglement entropy at finite temperature, as higher energy states become populated. 


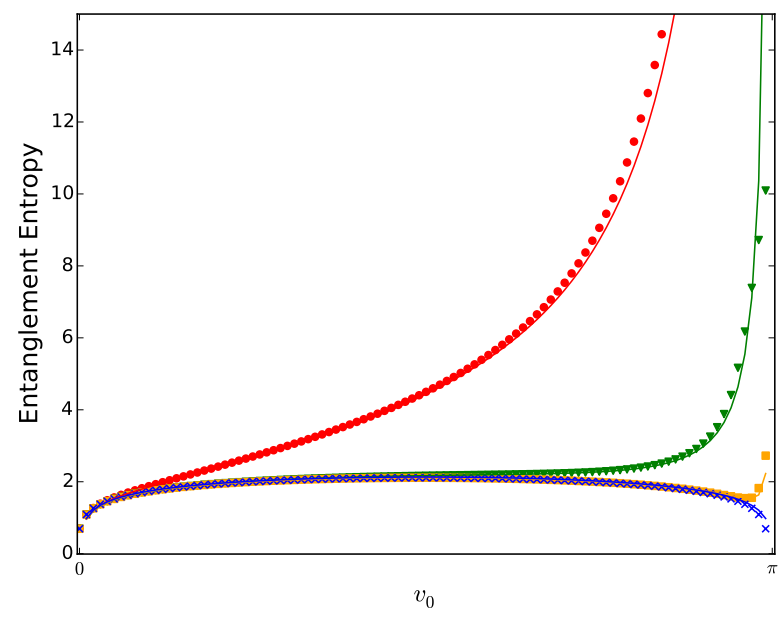

FIG. 5. Finite temperature entanglement entropy in the SSD model, for an interval centered about $v=\pi$, and for $\beta=$ $10,100,1000, \infty$ (from top to bottom). The dotted plots are numerical results from exact diagonalization and solid lines are the fitted analytic result (74) where we treat the nonuniversal constant part (independent of $v_{0}$ and $\beta$ ) as a fitting parameter.

\section{Dipolar limit}

The dipolar limit of our holographic SSD model (29) can be studied analogously to the holographic duals of the rainbow and SSD models.

a. Zero temperature A geodesic in these coordinates terminates at $u=0, v_{0}=-1 / x_{0}$, where $x_{0}=x(z=0)$ is the anchor point of the geodesic in the original Poincaré coordinates. We introduce a UV cutoff $\epsilon$ in the radial, $u$-direction. In terms of the boundary anchor point, the $z$ coordinate cutoff is

$$
\epsilon_{z}=\frac{a \epsilon}{v_{0}^{2}+\epsilon^{2}} \approx \frac{a \epsilon}{v_{0}^{2}} .
$$

Using (39) we have the following entanglement entropy:

$$
S_{A}\left(v_{0}\right)=\frac{c}{3} \log \left(\frac{2 v_{0}}{a \epsilon}\right) .
$$

As we can see, the holographic entanglement entropy is unchanged by the dipolar coordinate transformation in the bulk time slice.

The holographic result (77) can be readily reproduced from CFT calculations. From the previous subsection, we saw that the conformal boundary of the Poincaré metric in $u-v$ coordinates has the following metric:

$$
d s^{2}=-a^{-2} v^{4} d t^{2}+d v^{2} .
$$

Switching to imaginary time, $\tau$, and defining $x=-1 / v$, or metric can be written as a Weyl-transformed flat metric in the $x-\tau$ coordinates, $d s^{2}=e^{2 \sigma(x)} d s_{0}^{2}=e^{2 \sigma(x)} d \bar{z} d z$ where $z=x+i \tau$ and

$$
e^{2 \sigma(x)}=a^{-2} v^{4}=\frac{1}{a^{2} x^{4}}
$$

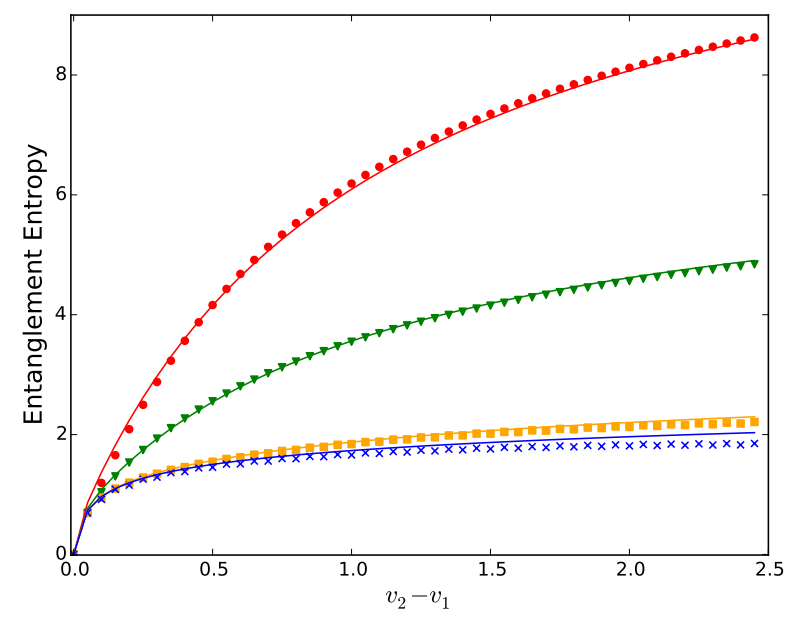

FIG. 6. Finite temperature entanglement entropy for the boundary theory of the dipole-foliated $A d S_{3}$ for an interval starting at $v=1.0$, for $\beta=1,2,10, \infty$ (from top to bottom). The plots are fit to (82). where $v_{1}$ and $v_{2}$ are the left and right boundaries of the interval, respectively, $c$ is the central charge, and $b$ is a fitting parameter related to the lattice cutoff. This is just the small $v$ limit of the function in Fig. 5 .

Using (51) as before, we can compute the correlator on this particular curved background:

$$
\begin{aligned}
\left.\operatorname{Tr}\left(\rho_{A}^{n}\right)\right|_{d s^{2}} & =c_{n} e^{-\Delta^{(n)}\left[\sigma\left(v_{1}\right)+\sigma\left(v_{2}\right)\right]}\left[\frac{1}{a \epsilon v_{1}}-\frac{1}{a \epsilon v_{2}}\right]^{-2 \Delta^{(n)}} \\
& =c_{n}\left[\frac{a \epsilon}{v_{2}-v_{1}}\right]^{-2 \Delta^{(n)}}
\end{aligned}
$$

Taking the log, multiplying by the appropriate factor, and taking the replica limit $n \rightarrow 1$, we recover (77).

b. Finite temperatures As for the entanglement entropy at finite temperatures, plugging the $v_{0}$-dependent, constant $u$, cutoff, $\frac{\epsilon}{v_{0}^{2}}$ into (49), we have

$$
S_{A}=\frac{c}{3} \log \left[\frac{\beta v_{0}^{2}}{\pi a \epsilon} \sinh \left(\frac{2 \pi a}{\beta v_{0}}\right)\right],
$$

which at any temperature, $1 / \beta$, asymptotes to the same logarithmic growth for large values of $v_{0}$. For generic endpoints, $v_{1}$ and $v_{2}$, we have

$$
S_{A}=\frac{c}{3} \log \left[\frac{\beta\left|v_{1} v_{2}\right|}{2 \pi a \epsilon} \sinh \left(\frac{2 \pi a}{\beta}\left|\frac{v_{2}-v_{1}}{v_{1} v_{2}}\right|\right)\right] .
$$

In Fig. 6, we compare (82) with numerical results obtained from the Hamiltonian (4) with $f_{j}=(10 j / N-5)^{2}$.

\section{CONCLUSION}

In this paper, we combined field theory, holographic, and numerical approaches, to investigate the entanglement entropy scaling for $(1+1) \mathrm{d}$ CFTs put on various 
inhomogeneous backgrounds. At both zero and finite temperatures, we confirmed that all of these approaches deliver consistent results.

While we focused on bipartite entanglement entropy, the (holographic) approach laid out in this work can be used to compute other, related quantities, such as mutual information and entanglement negativity. Contrary to bipartite entanglement entropy in CFTs, which is universal in the sense that it depends only on the central charge, these quantities depend on details (precise operator content) of CFTs, and can be used, e.g., to characterize quantum information scrambling. Negativity, in particular, could provide insight by drawing a clearer distinction between genuine quantum correlation and classical, thermal entropy in these curved systems; something that entanglement entropy misses. We are currently investigating negativity in $(1+1)$ d CFTs on inhomogeneous spaces.

Other interesting extensions of this work could involve applying our prescription to a wider varieties of inhomogeneous systems, and to far-from-non-equilibrium systems, such as systems undergoing quantum quenches ${ }^{22}$ and Floquet systems. ${ }^{23}$

\section{ACKNOWLEDGMENTS}

We thank Alexander Abanov, Jonah Kudler-Flam, Esperanza Lopez, Gautam Satishchandran, Hassan Shapourian, Tsukasa Tada, and Xueda Wen for useful discussions. SR is supported by a Simons Investigator Grant from the Simons Foundation. This work was supported in part by the National Science Foundation grant DMR 1455296.

\section{Appendix A: Application: Particles in a Potential Well}

The entanglement properties of nonrelativistic free fermions trapped in a potential well $V(x)$ can be described by a $c=1$ CFT on the following curved metric: ${ }^{28}$

$$
d s^{2}=d x^{2}-v^{2}(x) d t^{2}
$$

where $v(x)=\langle\rho(x)\rangle=\sqrt{\frac{2}{m}(\mu-V(x))}$. Here, as in Refs. 28 and 57 , we have used the "local density approximation". This is a semiclassical approximation in which the potential couples directly to the energy density, allowing for an easy application of our methods. Once we have this metric, we are implicitly using this approximation, and are no longer dealing directly with the nonrelativistic fermionic system. Henceforth, we use (A1) as our starting point for numerical and holographic calculations. Our holographic results are therefore not compared directly with the trapped fermion system, only with free fermions on the curved background given by (A1).

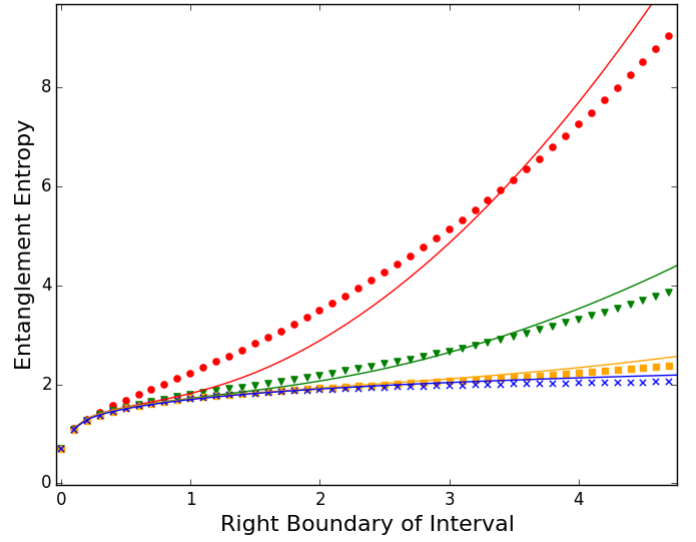

FIG. 7. Numerically computed entanglement entropy for free fermions in a Lorentzian potential well plotted at various temperatures, $\beta=10,30,100, \infty$ plotted in dotted lines. (A5) Plotted for the same temperatures in continuous lines.

Starting with the Poincaré metric on $A d S_{3}, d s_{A d S_{3}}^{2}=$ $\frac{1}{z^{2}}\left[d z^{2}+d y^{2}-d t^{2}\right]$, we can induce the metric (A1) on the conformal boundary with the appropriate coordinate transformation

$$
z=\frac{u}{v(x)}, \quad y=\int^{x} \frac{d x^{\prime}}{v\left(x^{\prime}\right)} .
$$

To leading order near the $u=0$ conformal boundary, our Poincaré metric takes the form

$$
d s^{2}=\frac{1}{u^{2}}\left[d u^{2}+d x^{2}-v(x)^{2} d t^{2}\right]+\mathcal{O}(1)
$$

Consider the example of a Lorentzian potential well, $V(x)=\frac{-1}{1+(x / a)^{2}}$, where $a$ controls the width of the well. We will set $\mu=0$. A lower chemical potential would result in an average particle density, $\langle\rho(x)\rangle$ that is zero outside of some finite region, requiring us to use BCFT methods. ${ }^{57}$ (Note, $\mu$ is not the chemical potential in our relativistic fermion system; it is better thought of as an input parameter for our metric). Our new coordinates are thus defined by (setting $m=2$ for convenience)

$$
\begin{aligned}
& z=u \sqrt{1+(x / a)^{2}}, \\
& y=\frac{1}{2}\left(x \sqrt{1+(x / a)^{2}}+a \sinh ^{-1}(x / a)\right)+\text { const. }
\end{aligned}
$$

This coordinate transformation implies a radial bulk cutoff of $\epsilon_{z}=\epsilon \sqrt{1+(x / a)^{2}}$. The finite temperature entanglement entropy for an interval between $-x_{0}$ and $x_{0}$ is thus

$$
\begin{aligned}
& S_{A}=\frac{c}{3} \log \left[\frac{\beta}{\pi \epsilon \sqrt{1+\left(x_{0} / a\right)^{2}}}\right] \\
& +\frac{c}{3} \log \left[\sinh \left(\frac{\pi}{\beta}\left(x_{0} \sqrt{1+\left(x_{0} / a\right)^{2}}+a \sinh ^{-1}\left(x_{0} / a\right)\right)\right)\right] .
\end{aligned}
$$


The zero temperature limit of (A5) is

$$
S_{A}=\frac{c}{3} \log \left(\frac{1}{\epsilon}\left(x_{0}+\frac{a \sinh ^{-1}\left(x_{0} / a\right)}{\sqrt{1+\left(x_{0} / a\right)^{2}}}\right)\right)
$$

In Fig. 7 we have plotted (A5) for several temperatures alongside numerical results for the Lorentzian well. The agreement is qualitatively quite good. The noticeable discrepancies are likely due to the limitations of the bulk transformation we have used, and the resulting $\mathcal{O}(1)$ contributions to the metric that we are ignoring. It should be noted once again that we have not directly simulated nonrelativistic fermions in a potential well; we have assumed that the metric (A1) is a valid desription of the physics in this potential, and simulated free fermions living on this background geometry.
1 G. Vitagliano, A. Riera, and J. I. Latorre. Volume-law scaling for the entanglement entropy in spin- $1 / 2$ chains. New Journal of Physics, 12(11):113049, November 2010.

${ }^{2}$ G. Ramírez, J. Rodríguez-Laguna, and G. Sierra. From conformal to volume law for the entanglement entropy in exponentially deformed critical spin $1 / 2$ chains. Journal of Statistical Mechanics: Theory and Experiment, 10:10004, October 2014.

3 G. Ramírez, J. Rodríguez-Laguna, and G. Sierra. Entanglement over the rainbow. Journal of Statistical Mechanics: Theory and Experiment, 6:06002, June 2015.

4 J. Rodríguez-Laguna, J. Dubail, G. Ramírez, P. Calabrese, and G. Sierra. More on the rainbow chain: entanglement, space-time geometry and thermal states. ArXiv e-prints, November 2016.

${ }^{5}$ V. Alba, S. N. Santalla, P. Ruggiero, J. Rodriguez-Laguna, P. Calabrese, and G. Sierra. Unusual area-law violation in random inhomogeneous systems. ArXiv e-prints, July 2018.

${ }^{6}$ E. Tonni, J. Rodríguez-Laguna, and G. Sierra. Entanglement hamiltonian and entanglement contour in inhomogeneous 1D critical systems. Journal of Statistical Mechanics: Theory and Experiment, 4:043105, April 2018.

7 A. Gendiar, R. Krcmar, and T. Nishino. Spherical Deformation for One-Dimensional Quantum Systems. Progress of Theoretical Physics, 122:953-967, October 2009.

8 T. Hikihara and T. Nishino. Connecting distant ends of one-dimensional critical systems by a sine-square deformation. Phys. Rev. B, 83(6):060414, February 2011.

9 A. Gendiar, M. Daniška, Y. Lee, and T. Nishino. Suppression of finite-size effects in one-dimensional correlated systems. Phys. Rev. A, 83(5):052118, May 2011.

10 N. Shibata and C. Hotta. Boundary effects in the densitymatrix renormalization group calculation. Phys. Rev. B, 84(11):115116, September 2011.

11 I. Maruyama, H. Katsura, and T. Hikihara. Sine-square deformation of free fermion systems in one and higher dimensions. Phys. Rev. B, 84(16):165132, October 2011.

${ }^{12} \mathrm{H}$. Katsura. Exact ground state of the sine-square deformed XY spin chain. Journal of Physics A Mathematical General, 44(25):252001, June 2011.

13 H. Katsura. Sine-square deformation of solvable spin chains and conformal field theories. Journal of Physics A Mathematical General, 45(11):115003, March 2012.

14 C. Hotta and N. Shibata. Grand canonical finite-size numerical approaches: A route to measuring bulk properties in an applied field. Phys. Rev. B, 86(4):041108, July 2012.

${ }^{15}$ K. Okunishi and H. Katsura. Sine-square deformation and supersymmetric quantum mechanics. Journal of Physics A Mathematical General, 48:445208, November 2015.
16 T. Tada. Sine-square deformation and its relevance to string theory. Modern Physics Letters A, 30:1550092, May 2015.

17 N. Ishibashi and T. Tada. Infinite circumference limit of conformal field theory. Journal of Physics A Mathematical General, 48:315402, August 2015.

18 K. Okunishi. Sine-square deformation and Mobius quantization of two-dimensional conformal field theory. ArXiv e-prints, March 2016.

19 N. Ishibashi and T. Tada. Dipolar quantization and the infinite circumference limit of two-dimensional conformal field theories. International Journal of Modern Physics A, 31:1650170, November 2016.

20 X. Wen, S. Ryu, and A. W. W. Ludwig. Evolution operators in conformal field theories and conformal mappings: Entanglement Hamiltonian, the sine-square deformation, and others. Phys. Rev. B, 93(23):235119, June 2016.

21 S. Tamura and H. Katsura. Zero-energy states in conformal field theory with sine-square deformation. ArXiv e-prints, September 2017.

${ }^{22} \mathrm{X}$. Wen and J.-Q. Wu. Quantum dynamics in sine-square deformed conformal field theory: Quench from uniform to nonuniform conformal field theory. Phys. Rev. B, 97(18):184309, May 2018.

23 X. Wen and J.-Q. Wu. Floquet conformal field theory. ArXiv e-prints, April 2018.

24 T. Tada. Conformal quantum mechanics and sine-square deformation. Progress of Theoretical and Experimental Physics, 2018(6):061B01, June 2018.

25 Peter D. Hislop and Roberto Longo. Modular structure of the local algebras associated with the free massless scalar field theory. Communications in Mathematical Physics, 84(1):7185, 1982.

26 I. Peschel. On the entanglement entropy for an XY spin chain. Journal of Statistical Mechanics: Theory and Experiment, 12:12005, December 2004.

27 J. Cardy and E. Tonni. Entanglement Hamiltonians in twodimensional conformal field theory. Journal of Statistical Mechanics: Theory and Experiment, 12:123103, December 2016.

28 J. Dubail, J.-M. Stéphan, J. Viti, and P. Calabrese. Conformal Field Theory for Inhomogeneous One-dimensional Quantum Systems: the Example of Non-Interacting Fermi Gases. ArXiv e-prints, June 2016.

29 S. Murciano, P. Ruggiero, and P. Calabrese. Entanglement and relative entropies for low-lying excited states in inhomogeneous one-dimensional quantum systems. ArXiv e-prints, October 2018.

30 H. Ueda, A. Gendiar, V. Zauner, T. Iharagi, and T. Nishino. Transverse Field Ising Model Under Hyper- 
bolic Deformation. ArXiv e-prints, August 2010.

31 H. Ueda, H. Nakano, K. Kusakabe, and T. Nishino. Scaling Relation for Excitation Energy under Hyperbolic Deformation. Progress of Theoretical Physics, 124:389-398, September 2010.

32 T. Iharagi, A. Gendiar, H. Ueda, and T. Nishino. Phase Transition of the Ising Model on a Hyperbolic Lattice. Journal of the Physical Society of Japan, 79(10):104001104001, October 2010.

33 E. Langmann and P. Moosavi. Diffusive heat waves in random conformal field theory. ArXiv e-prints, July 2018.

34 J. Dubail, J.-M. Stéphan, and P. Calabrese. Emergence of curved light-cones in a class of inhomogeneous Luttinger liquids. ArXiv e-prints, May 2017.

35 O. Aharony, D. Marolf, and M. Rangamani. Conformal field theories in anti-de Sitter space. Journal of High Energy Physics, 2:41, February 2011.

${ }^{36}$ K. Jensen, A. Karch, and B. Robinson. Holographic dual of a Hawking pair has a wormhole. Phys. Rev. D, 90(6):064019, September 2014.

${ }^{37}$ T. Andrade and C. F. Uhlemann. Beyond the unitarity bound in $\operatorname{AdS} / \mathrm{CFT}_{(A) d S}$. Journal of High Energy Physics, 1:123, January 2012.

${ }^{38}$ K. Skenderis and S. N. Solodukhin. Quantum effective action from the AdS/CFT correspondence. Physics Letters B, 472:316-322, January 2000.

39 M. Parikh and P. Samantray. Rindler-AdS/CFT. ArXiv e-prints, November 2012.

40 B. D. Chowdhury and M. K. arikh. When UV and IR Collide: Inequivalent CFTs From Different Foliations Of AdS. ArXiv e-prints, July 2014.

41 S. Deser and O. Levin. LETTER TO THE EDITOR: Equivalence of Hawking and Unruh temperatures and entropies through flat space embeddings. Classical and Quantum Gravity, 15:L85-L87, December 1998.

42 E. Cáceres, M. Chernicoff, A. Güijosa, and J. F. Pedraza. Quantum Fluctuations and the Unruh effect in stronglycoupled conformal field theories. Journal of High Energy Physics, 6:78, June 2010.

${ }^{43}$ H. Casini, M. Huerta, and R. C. Myers. Towards a derivation of holographic entanglement entropy. Journal of High Energy Physics, 5:36, May 2011.

44 T. Hirayama, P.-W. Kao, S. Kawamoto, and F.-L. Lin. Unruh effect and holography. Nuclear Physics B, 844:1-
25, March 2011.

45 T. Takayanagi. Holographic Dual of a Boundary Conformal Field Theory. Physical Review Letters, 107(10):101602, September 2011.

46 M. Fujita, T. Takayanagi, and E. Tonni. Aspects of AdS/BCFT. Journal of High Energy Physics, 11:43, November 2011.

47 M. Bañados. Three-dimensional quantum geometry and black holes. In H. Falomir, R. E. Gamboa Saravi, and F. A. Schaposnik, editors, Trends in Theoretical Physics II, volume 484 of American Institute of Physics Conference Series, pages 147-169, July 1999.

48 M. M. Roberts. Time evolution of entanglement entropy from a pulse. Journal of High Energy Physics, 12:27, December 2012.

49 G. Mandal, R. Sinha, and N. Sorokhaibam. The inside outs of $\mathrm{AdS}_{3} / \mathrm{CFT}_{2}$ : exact AdS wormholes with entangled CFT duals. Journal of High Energy Physics, 1:36, January 2015.

50 M. Flory and N. Miekley. Complexity change under conformal transformations in AdS_3/CFT_2. ArXiv e-prints, June 2018.

51 D. Anninos, J. Samani, and E. Shaghoulian. Warped entanglement entropy. Journal of High Energy Physics, 2:118, February 2014.

52 S. Ryu and T. Takayanagi. Aspects of holographic entanglement entropy. Journal of High Energy Physics, 8:045, August 2006.

53 S. Ryu and T. Takayanagi. Holographic Derivation of Entanglement Entropy from the anti de Sitter Space/Conformal Field Theory Correspondence. Physical Review Letters, 96(18):181602, May 2006.

54 V. E. Hubeny, M. Rangamani, and T. Takayanagi. A covariant holographic entanglement entropy proposal. Journal of High Energy Physics, 7:062, July 2007.

55 M. M. Sheikh-Jabbari and H. Yavartanoo. Excitation entanglement entropy in two dimensional conformal field theories. Phys. Rev. D, 94(12):126006, December 2016.

${ }^{56} \mathrm{~N}$. Tetradis. Entropy from $\mathrm{AdS}_{3} / \mathrm{CFT}_{2}$. Journal of High Energy Physics, 2:54, February 2012.

57 Y. Brun and J. Dubail. One-particle density matrix of trapped one-dimensional impenetrable bosons from conformal invariance. ArXiv e-prints, January 2017. 